\title{
Antimicrobial Susceptibility Pattern of Community Acquired Pneumonia among Adults in Tripura, North-Eastern India
}

\author{
Dr. Mousumi Paul ${ }^{1 *}$, Dr. Tapan Majumdar², Dr. Pradip Bhowmik ${ }^{3}$
}

\author{
${ }^{1}$ Medical Officer-MD Microbiology, Department of Microbiology-Tripura Health Service, Agartala Government Medical College \& GBP Hospital, \\ Agartala, Pin no. 799006, Tripura \\ ${ }^{2}$ Professor and Head, Department of Microbiology, Agartala Government Medical College \& GBP Hospital, Agartala, Pin no. 799006, Tripura \\ ${ }^{3}$ Professor, Department of Medicine, Agartala Government Medical College \& GBP Hospital, Agartala, Pin no. 799006, Tripura
}

DOI: $10.36347 /$ sjams.2020.v08i08.022

| Received: 18.08.2020 | Accepted: 25.08.2020 | Published: 28.08.2020

*Corresponding author: Dr. Mousumi Paul

Abstract

Original Research Article

Community Acquired Pneumonia (CAP) is the most common respiratory tract infection in day to day practice. The knowledge of organism commonly causative of CAP helps in early empirical treatment initiation. The true incidence of pneumonia acquired in the community is unknown, but this is a common clinical problem worldwide especially in developing countries and remains a leading cause of death in India. There are very few and conflicting Indian data regarding the bacteriological etiology of community acquired pneumonia (CAP). Adding to this agony, there is no much credible data from the north-eastern part of India. Hence the following study was undertaken to study the aerobic bacteriological profile and antimicrobial susceptibility pattern of community-acquired pneumonia among adult. A total of 220 patients were studied. Age group varied from 18 years to 85 years. Most common organisms responsible were Klebsiella pneumonia, Staphylococcus aureus, Pseudomonas aeruginosa, Haemophilus influenzae, Streptococcus pneumoniae. Gram negative Organisms were found to be sensitive for Meropenem (100\%), followed by PiperacillinTazobactum (87.95\%), Amikacin (84.34\%), Ceftriaxone (73.49\%) and Levofloxacin (69.88\%). Highest number of resistance was seen against Amoxycillin-Clavulanic acid (74.39\%), followed by Ceftazidime (66.67). For gram positive cocci, highest sensitivity was shown to Clindamycin $(83.67 \%)$, followed by Doxycycline $(81.63 \%) \&$ Ceftriaxone (73.47\%), Conclusion: Klebsiella pneumoniae was the most common pathogen incriminated in CAP, followed by Staphylococcus aureus. Most of the organisms were found to be sensitive to carbapenem, third generation cephalosporins, fluroquinolones, macrolides.

Keywords: North-eastern india, Community acquired pneumonia, antimicrobial susceptibility pattern, bacteriological profile.

Copyright @ 2020: This is an open-access article distributed under the terms of the Creative Commons Attribution license which permits unrestricted use, distribution, and reproduction in any medium for non-commercial use (NonCommercial, or CC-BY-NC) provided the original author and source are credited.

\section{INTRODUCTION}

Pneumonia is defined as an acute respiratory illness associated with recently developed radiological pulmonary shadowing which may be segmental, lobar or multilobar. Pneumonia is usually classified as Community Acquired Pneumonia (CAP) and Hospital acquired pneumonia (HAP) [1].

Infectious Diseases Society of America (IDSA) defines CAP as "an acute infection of the pulmonary parenchyma that is associated with at least some symptoms of acute infection, accompanied by the presence of an acute infiltrate on a chest radiograph or auscultatory findings consistent with pneumonia in a patient not hospitalized or residing in a long-term care facility for more than 14 days before onset of symptoms" [2]. Around $20 \%$ of the mortality due to infectious diseases in India is caused by lower respiratory tract infections among which pneumonia is the most important culprit [3]. Bacteriological profile in community acquired pneumonia (CAP) is different in different countries and changes with time even within the same country. Looking at world literatures microbiological diagnosis could be made in only 40$71 \%$ of cases of CAP even when extensive methods have been used when compared to $>90 \%$ cases in preantibiotic era [3]. The widespread antibiotic (mis) use is probably responsible for decreasing culture rates in CAP. The problem of pneumonia is much greater in developing countries where pneumonia is the most common cause of hospital attendance in adults [4]. Sir William Osler, known as "the father of modern medicine," appreciated the morbidity and mortality of pneumonia, describing it as the "captain of the men of death" in 1918. Pneumonia is a common illness 
affecting approximately 450 million people a year and occurring in all parts of the world. It is a major cause of death among all age groups resulting in 4 million deaths (7\% of the world's total death) yearly [5].

As many as 4 million cases of CAP occur annually and $20 \%$ of them require hospitalization. The mortality ranges from $13.6 \%$ in hospitalized patients to $36.5 \%$ in patients admitted to ICU [6].

In India estimated death rate per 100,000 population in 2004 was $89.5 \%$ due to LRTI \& estimated disability adjusted life years (DALYs) per 100,000 population in 2004 was 1894 [7].

Aetiology of CAP is generally bacterial but the microbial pattern varies from country to country. It varies with time and geographical distribution within the same country and so does the antimicrobial sensitivity and emerging resistance pattern. Streptococcus pneumoniae is the most common etiological agent in United Kingdom, Europe, United States of America and Iraq. In India Streptococcus pneumoniae is most common causative organism of pneumonia in Delhi, Shimla, Ludhiana and Pune [8, 9]. Staphylococcus aureus is the most common organism isolated in CAP at Manipur, India [10].

The treatment of CAP is complicated by growing threat of antimicrobial resistance and the tendency to rely on empirical therapy. Indiscriminate use of antibiotics has led us to emergence of multidrug resistant strains. The scenario is getting worsen day by day. Recent years have witnessed the emergence of new pathogens and also no newer antibiotics designed to combat them [11].

Most of organism are developing resistance to various antibiotics, now a day. The organism is mainly sensitive to third generation cephalosporins, fluroquinolones and aminoglycosides [7]. The problem of resistance is leading to increased morbidity and mortality by increasing duration of hospital stay, rendering our therapy ineffective and by increasing the complications [12].

Although a wide variety of recognized pathogens cause CAP, the precise etiology, pattern of microbial flora in various settings, antibiotic sensitivity and resistance in India is still not comprehensively studied [13].

Empirical therapy should be guided by the antibiogram of a study conducted in a tertiary care hospital. The life threatening outcome of pneumonia underscores the importance of use of timely surveillance data to guide the effective choice of empirical therapy which will have a great impact on mortality \& morbidity of patients.
In view of the above observations made by various workers, the present study is being conducted to know the causative agents of the CAP and to find out the antibiotic sensitivity pattern (antibiogram) of the causative organisms so that the patients are properly diagnosed and treated with specific antibiotics. Further, no similar studies has been carried out in this state. This study will also give us information regarding the occurrence, types of the causative bacterial agents their antibiogram, \& socio-demographic profile of adult onset CAP patients in a tertiary care hospital in this state. Lastly the study will have an impact on the overall understanding \& management of adult onset CAP \& to reduce the mortality and morbidity of pneumonia in this state.

\section{Material And Methods}

The present study was conducted at Department of Microbiology in collaboration with the Department of Medicine of AGMC \& GBP Hospital between December 2017 and June 2019. This study is a hospital based cross sectional study carried out on 220 cases of community-acquired pneumonia of patients aged $\geq 18$ years. Prior to the study, the protocol was approved by the institutional ethical committee, and all patients gave their informed consent to participate.

Inclusion criteria were subjects aged $\geq 18$ years old, outpatients with symptoms, signs and laboratory data diagnostic of pneumonia as well as inpatients with pneumonia at the time of hospitalization. Patients who had already received antibiotics before sputum could be sent for culture sensitivity, aspiration pneumonia, obstructive pneumonia, immune-compromised state, nosocomial pneumonia were excluded from the study

Sputum and bronchoalveolar lavage was collected for gram stain and culture and sensitivity, before starting empirical antibiotic therapy. Following gram staining those specimens were inoculated in Blood agar (BA), MacConkey agar (MA), Chocolate agar (CA). Inoculation in chocolate agar was done when pus cell count in direct gram staining was observed to be more than 50/hpf. The inoculated plates were incubated aerobically at $37^{\circ} \mathrm{C}$ overnight. The inoculated chocolate agar \& sheep blood agar suspecting growth for Streptococcus pneumoniae however were incubated at $5-10 \% \quad \mathrm{CO}_{2}$ (candle jar) with similar duration and temperature.

\section{RESULTS}

The results and observations of the study are as follows Distribution of sample: During the study period 216 sputum samples and 4 broncoalveolar lavage samples were collected from a total of 220 study population (comprising of $122(55.45 \%)$ from IPD,75(34.10\%) from OPD \& 23(10.45\%) from ICU) of community acquired pneumonia as per inclusion and exclusion criteria from medicine \& chest department. 
Distribution of study population according to severity score (CURB-65): 145 samples were collected from patients admitted in hospital (IPD \& ICU) based on their pneumonia severity score - CURB 65 (Figure$1)$.

Sputum Microscopical examination: The sputum smears were gram stained and examined under microscope for determination of their adequacy. Out of 216 sputum samples, 165 samples showed > 10 pus cells/epithelial cells under low power field in gram staining (Figure $2 \& 3$ ). Rest 51 sputum samples were recollected from patients and the adequacy of samples were revalidated. After validation of sputum samples bacteriological culture was done. Of those smears predominate organisms were, $39(18.06 \%)$ gram positive cocci, $70(32.41 \%)$ gram-negative bacilli, and $107(49.54 \%)$ were mixed. Details are shown in Table1.

Bacteriological culture and isolation: Out of 216 sputum samples, $127(58.80 \%)$ were found to be culture positive for bacterial isolates \& respiratory commensals grew in $89(41.20 \%)$ samples. Among the 127 culture positive samples, $81(63.78 \%)$ and $46(36.22 \%)$ were GNB and GPC respectively. Out of the 4 BAL samples, all $(100 \%)$ were found to be culture positive, $1(25 \%)$ being GNB \& the remaining $3(75 \%)$ were GPC.

The most common organism isolated from sputum culture was Klebsiella pneumoniae $(\mathrm{n}=44$,
20.4\%), followed by Staphylococcus aureus ( $\mathrm{n}=35$, 16.2\%), Pseudomonas aeruginosa $(\mathrm{n}=18,8.3 \%)$, Streptococcus pneumoniae $(\mathrm{n}=9,4.2 \%)$, Citrobacter koseri $(\mathrm{n}=9,4.2 \%)$, Escherichia coli $(\mathrm{n}=5,2.3 \%)$, Citrobacter freundii $(\mathrm{n}=3,1.4 \%)$, Streptococcus pyogenes $(\mathrm{n}=2,0.9 \%)$, Haemophilus influenzae $(\mathrm{n}=2$, $1 \%$ ), whereas the 4 BAL samples comprised of Klebsiella pneumoniae $(\mathrm{n}=1,25 \%)$ \& Staphylococcus aureus $(\mathrm{n}=3,75 \%)$ (Figure-4).

**Sputum gram staining and culture demonstrated correlation in 109 of 216 patients (with both gram staining and culture positivity and 89 patients had respiratory commensal grown in culture)

Antibiotic sensitivity testing: Out of 82 GNB isolated from sputum \& BAL samples $(81 \& 1$ respectively), $100 \%$ isolates were sensitive to Meropenem, followed by Piperacillin-Tazobactum (87.95\%), Amikacin $(84.34 \%)$, Ceftriaxone $(73.49 \%)$ and Levofloxacin (69.88\%). Highest number of resistance was seen against Amoxicillin-clavulanic acid $(74.39 \%)$, followed by Ceftazidime $(66.67 \%)$ (Table-2).

For GPC (46 from sputum samples \& 3 from BAL samples), highest sensitivity was shown to Clindamycin $(83.67 \%)$, followed by Doxycycline $(81.63 \%)$ \& Ceftriaxone $(73.47 \%)$, whereas maximum resistance was seen against Erythromycin (57.14\%), followed by Amoxyclav (34.69\%) (Table-3).

Table-1: Sputum gram staining findings

\begin{tabular}{|l|l|l|}
\hline Gram staining (predominate organism) & No. of patients & Percentage (\%) \\
\hline Gram positive cocci & 39 & 18.06 \\
\hline Gram negative bacilli & 70 & 32.41 \\
\hline Mixed organisms & 107 & 49.54 \\
\hline
\end{tabular}

Table-2: AST of various GNB

\begin{tabular}{|l|l|l|l|l|l|l|}
\hline \multirow{2}{*}{ Antibiotics } & \multicolumn{6}{l}{ Organisms (Percentage of sensitivity) } \\
\cline { 2 - 7 } & $\begin{array}{l}\text { K.pneumoniae } \\
(\mathrm{n}=45)\end{array}$ & $\begin{array}{l}\text { P.aeruginosa } \\
(\mathrm{n}=18)\end{array}$ & $\begin{array}{l}\text { C.koseri } \\
(\mathrm{n}=9)\end{array}$ & $\begin{array}{l}\text { E.coli } \\
(\mathrm{n}=5)\end{array}$ & $\begin{array}{l}\text { C.freundii } \\
(\mathrm{n}=3)\end{array}$ & $\begin{array}{l}\text { H.influenzae } \\
(\mathrm{n}=2)\end{array}$ \\
\hline AMC & $26.67 \%(12)$ & $22.22 \%(4)$ & $33.33 \%(3)$ & $20 \%(1)$ & $33.33 \%(1)$ & $100 \%(2)$ \\
\hline AK & $84.44 \%(38)$ & $88.86 \%(16)$ & $88.89 \%(8)$ & $100 \%(5)$ & $66.67 \%(2)$ & - \\
\hline CTR & $75.56 \%(34)$ & $72.22 \%(13)$ & $88.89 \%(8)$ & $60 \%(3)$ & $66.67 \%(2)$ & - \\
\hline LEV & $71.11 \%(32)$ & $66.67 \%(12)$ & $77.78 \%(7)$ & $40 \%(2)$ & $33.33 \%(1)$ & $100 \%(2)$ \\
\hline PIT & $88.89 \%(40)$ & $94.44 \%(17)$ & $100 \%(9)$ & $60 \%(3)$ & $100 \%(3)$ & - \\
\hline MRP & $100 \%(45)$ & $100 \%(18)$ & $100 \%(9)$ & $100 \%(5)$ & $100 \%(3)$ & - \\
\hline CAZ & - & $33.33 \%(6)$ & - & - & - & $100 \%(2)$ \\
\hline CLR & - & - & - & - & - & $50 \%(1)$ \\
\hline CHL & - & - & - & - & - & $100 \%(2)$ \\
\hline
\end{tabular}


Table-3: AST of various GPC

\begin{tabular}{|l|l|l|l|}
\hline \multirow{2}{*}{ Antibiotics } & \multicolumn{3}{|l|}{ Organisms (percentage of sensitivity) } \\
\cline { 2 - 4 } & Staphylococcus aureus $(\mathbf{n = 3 8})$ & Streptococcus pneumoniae $(\mathbf{n}=9)$ & Streptococcus pyogenes $(\mathbf{n = 2})$ \\
\hline AMC & $57.89 \%(22)$ & $88.89 \%(8)$ & $0 \%(0)$ \\
\hline CD & $81.58 \%(31)$ & $88.89 \%(8)$ & $0 \%(0)$ \\
\hline LEV & $57.89 \%(22)$ & $88.89 \%(8)$ & $0 \%(0)$ \\
\hline E & $42.11 \%(16)$ & $44.44 \%(4)$ & $50 \%(1)$ \\
\hline DO & $89.47 \%(34)$ & $55.56 \%(5)$ & $50 \%(1)$ \\
\hline CTR & $73.68 \%(28)$ & $77.78 \%(7)$ & $50 \%(1)$ \\
\hline
\end{tabular}

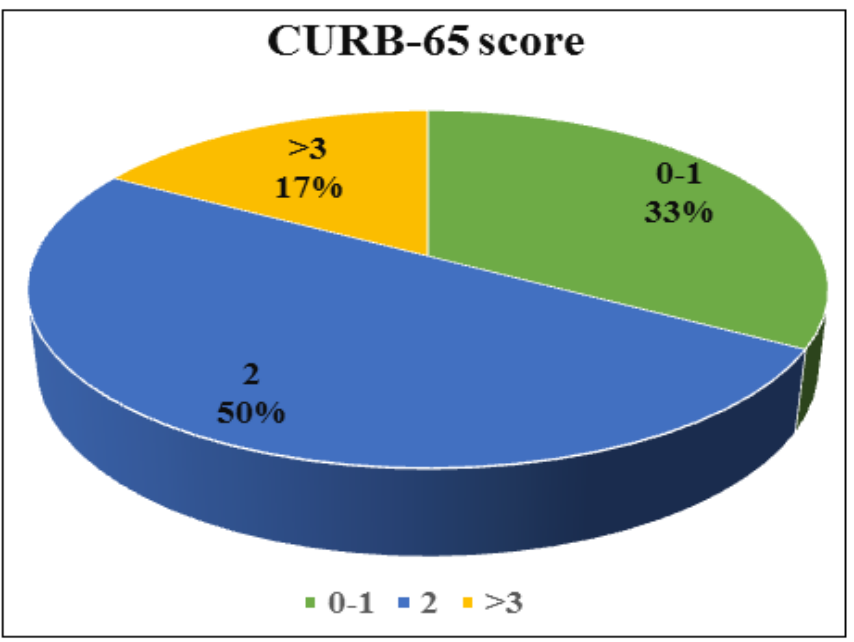

Fig-1: Pie chart showing distribution of CURB 65 Score of the study population

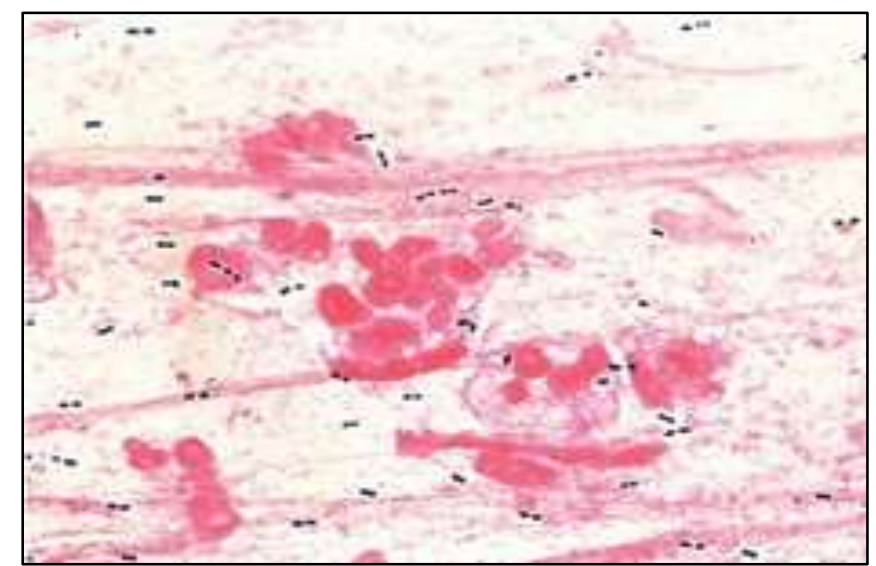

Fig-2: Gram staining of sputum sample showing gram positive cocci in pair)

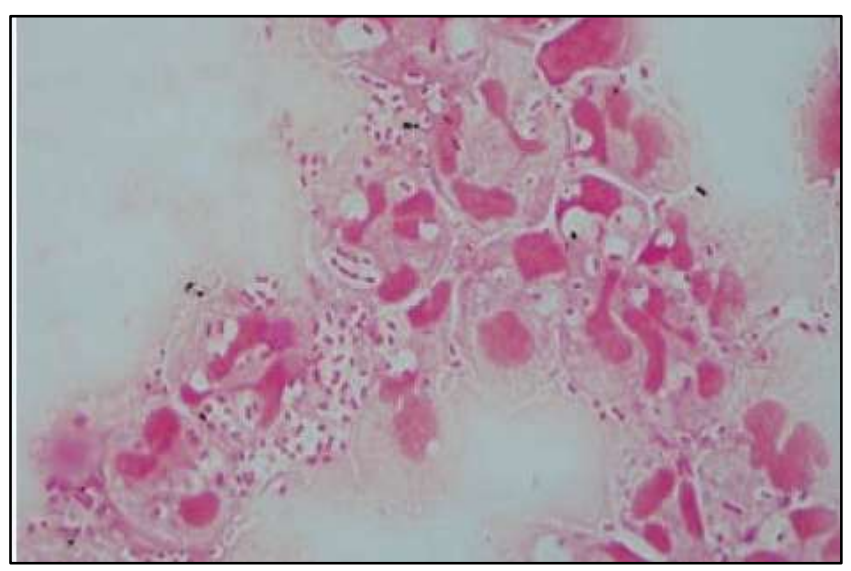

Fig-3: Gram staining of sputum sample showing gram negative bacilli 


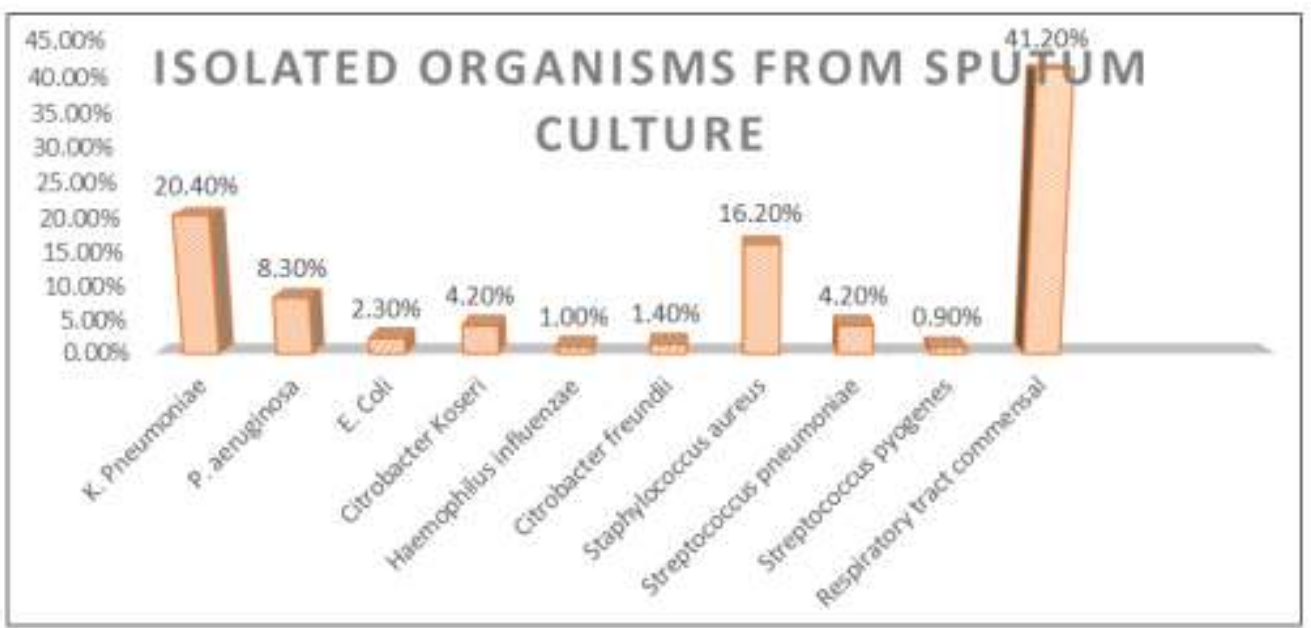

Fig-4: Bar diagram showing distribution of study population according to isolated organisms from sputum culture

\section{DISCUSSION}

CAP is a frequent cause of hospital admission and mortality in elderly patients worldwide. The clinical presentation, etiology, and outcome of communityacquired pneumonia in elderly differs from that of other population. In the present study, 80 patients of community-acquired pneumonia $>60$ years of age were included. The results of this study which has been presented in previously are discussed below.

Study population distribution: In our study 216 sputum samples and 4 bronco-alveolar lavage samples were collected from a total of 220 study population (comprising of $122(55.45 \%)$ from IPD, $75(34.10 \%)$ from OPD \& 23(10.45\%) from ICU) of CAP as per inclusion and exclusion criteria from medicine \& chest department- which indicates a large number of CAP patients need hospitalization and even ICU admission.

According to one eastern Indian study conducted by Khadanga $\mathrm{S}$ et al., all of their CAP patients (464 no.) were hospitalized, among them ICU admission rate was $16.1 \%$ [14]. Our result coincide with their patient distribution.

Sputum Microscopy and Culture: In this study, the microbial diagnosis of CAP was confirmed by sputum culture only in $58.80 \%$ patients. This result is corresponding with Jain et al. study (45.8\%) [15]. The overall rate of identification of microbial aetiology in other parts of India: $75.6 \%$ in Shimla [16], $47.7 \%$ in Chandigarh [17], or other parts of world $62 \%$ in UK [18], 68\% in Singapore [19] and 56\% in Philippines [20]. This could be due to the limited use of laboratory tests. In this study, we used sputum, BAL culture and microscopy as diagnostic tools to identify the aerobic bacterial pathogen causing pneumonia. Serology for both atypical and viral pathogens was not done at the time of this study.

In this study $127(58.80 \%)$ pathogens were isolated by sputum culture. But this is another fact that even with the use of extensive laboratory testing and various invasive procedures in different studies, etiological confirmation could be achieved only in 45 $75 \%$ patients $[4,15-18,21,22]$.

Decreased sputum positivity is due to prior use of antibiotics, inappropriate sputum production and non-productive cough.

Streptococcus pneumoniae has been identified as the commonest organism causing CAP all over the world [14, 23-30]. But some studies, over the last three decades, have reported higher incidence of gramnegative organisms among culture- positive pneumonias $[4,31]$. Most of the patients from whom gram-negative bacteria was isolated were over 50 years of age, smokers or had COPD. It has been reported that old age, smoking and COPD impair pulmonary defences and pre-dispose to CAP caused by gram-negative bacteria.

In this study most commonly isolated pathogen was Klebsiella pneumoniae accounting for $20.4 \%$. Next common was Staphylococcus aureus which accounts for $16.20 \%$ this was followed by other Gram-negative bacilli - Pseudomonas aeruginosa $8.30 \%$, Citrobacter koseri $4.20 \%$, Escherichia coli $2.30 \%$, Citrobacter freundii $1.40 \%$ and Haemophilus influenzae $1 \%$. Streptococcus pneumoniae and Streptococcus pyogenes was isolated in $4.20 \%$ and $0.9 \%$ of cases respectively. As per some Indian studies, over last three decades have reported higher incidence of Gram-negative organisms among culture positive community acquired pneumonia cases $[4,9,15,31,33,34]$.

The second commonest organism isolated from sputum culture was staphylococcus aureus. The high incidence of staphylococcus in CAP can be explained by spread of staphylococcus from hospital setting to community and staphylococcus complicating virus illness esp. influenza $[9,10]$. 
Mousumi Paul et al., Sch J App Med Sci, August, 2020; 8(8): 1901-1907

Antibiotic sensitivity testing: The role of the microbiology laboratory in the diagnosis of CAP remains controversial. As per Gupta et al., [35] National pneumonia guidelines, yield of sputum culture varies from $34 \%$ to $86 \%$.In our study, organism was found in $58.80 \%$ of sputum culture reports; it is still recommend sending a routine sputum culture with Gram stain to optimize antibiotic therapy for each individual patient as well as to monitor for drug-resistance among pathogens.

Choosing the proper antibiotics as initial empiric therapy \& later streamlining as per the culture sensitivity pattern is critical in outcome of CAP. Important considerations include penetration into respiratory secretions, spectrum of activity and antimicrobial resistance. These factors limit the usefulness of drugs such as amoxicillin, erythromycin and trimethoprim-sulfamethoxazole.

Woodhead et al., [36] in a study found that in non-severe CAP oral $\beta$ lactam antibiotics, macrolides, or fluoroquinolones are equally effective when judged by clinical cure and mortality. They recommended that $\beta$ lactam antibiotic (with macrolides and tetracyclines as good alternatives in individuals who are hypersensitive to penicillin) should usually remain the preferred therapy for patients with non-severe community acquired pneumonia managed in the community or in hospital and among $\beta$ lactam antibiotics, as oral cephalosporins have poor pharmacokinetics it would seem that amoxicillin or amoxicillin-clavulanate should usually be the first choice for therapy.

In our study population most of them showed good response to injectable 3rd generation cephalosporins or macrolides or in combination. However sensitivity pattern among the patients with sputum positivity for gram negative bacilli showed meropenem, amikacin and piperacillin-tazobactum as better sensitivity compared to others, and for gram positive cocci clindamycin, doxycycline, ceftriaxone are the drug of choice. However considering the age and risk of renal impairment still 3rd generation cephalosporins and doxycycline will be considered superior.

\section{CONCLUSION}

Klebsiella pneumoniae was the most common pathogen incriminated in CAP, followed by Staphylococcus aureus. But in general the emergence of the higher incidence of Gram-negative organism especially Klebsiella pneumoniae has occurred in our geographical area.

In our study we found the most effective antibiotics for gram negative bacilli causing CAP was meropenem followed by piperacillin-tazobactum and amikacin, and for gram positive cocci was ceftriaxone, clindamycin \& levofloxacin. So these drugs can be started as an empirical therapy for treatment of CAP and thereafter according to the antibiotic susceptibility testing report, escalation and de-escalation can be done.

\section{Source of Support: Nil}

Conflict of Interest: Nil

\section{REFERENCE}

1. Reid PT, Innes JA. Respiratory disease. In: Colledge NR, Walker BR, Ralston SH, editors. Davidson's Principles \& Practice of Medicine. $21^{\text {st }}$ edition. New York: Elsevier; 2010; 680-8.

2. Mandell LA, Wunderink RG, Anzueto A, Bartlett JG, Campbell GD, Dean NC, Dowell SF, File TM, Daniel MM, Michael SN, Torres A, Whitney CG. Infectious diseases society of America/American Thoracic Society consensus guidelines on the management of community acquired pneumonia in adults. Clin Infect Dis. 2007;44(Supplement 2):S27-72.

3. Khadanga S, Tadepalli K,Thatoi PK, Behera SK. Changing bacteriological profile and mortality trends in community acquired pneumonia. J Glob Infect Dis. 2014;6(4):186-8.

4. Chintaman AC, Ghadage DP, Bhore AV. Bacteriological profile of community acquired pneumonia in a tertiary care hospital. Int J Curr Microbiol App Sci. 2017;6(4):190-4.

5. Kejriwal A, Shenoi AS, Pusukuru R, Sebastien C, Bhutta K. A clinical, bacteriological and radiological profile of community acquired pneumonia in Navi Mumbai, India. IOSR-JDMS. 2015;14(9):58-61.

6. Silla M, Babu GR, Rani NU. Clinical, etiological profile and outcome of hospitalized patients of community acquired pneumonia. IJBAMR. 2017;6(3):576-85.

7. Epidemiology of community acquired pneumonia. Supplement to Journal of the Association of Physicians of India. 2013;61:7-8.

8. Kotwani A, Kumar S, Swain PK, Suri JC, Gaur SN. Antimicrobial drug prescribing patterns for community-acquired pneumonia in hospitalized patients: A retrospective pilot study from New Delhi, India. Indian J Pharmacol. 2015;47(4):37582.

9. Shah BA, Singh G, Naik MA, Dhobi GN. Bacteriological and clinical profile of community acquired pneumonia in hospitalised patients. Lung India. 2010;27(2):54-7.

10. Vanlalruati RSC, Devi M, Singh NB, Singh NT. A study of bacteriological profile (aerobic) and antimicrobial susceptibility of community acquired pneumonia cases. J Commun Dis. 2012;44(1):479.

11. Ali MK, Batchoun RG, Nour TM. Etiology of community-acquired pneumonia in hospitalized patients in Jordan. Saudi Med J. 2006;27:813-6. 
12. Savaliya A, Sodavadiya KB, Mangukiya KK. Study of clinical and bacteriological profile of community acquired pneumonia (cap) and its complications. I.J.S.N. 2013;4(4):702-10.

13. Acharya VK, Padyana M, Unnikrishnan B, Anand R, Acharya PR, Juneja DJ. Microbiological profile and drug sensitivity pattern among community acquired pneumonia patients in a tertiary care center in Mangalore, Coastal Karnataka, India. Journal of Clinical and Diagnostic Research. 2014;8(6):MC04-MC06.

14. Sandeep KJ, Shiping J, Shushma T. Study of clinical, radiological and bacteriological profile of community acquired pneumonia in hospitalized patients of Gajra Raja Medical College,Gwalior. International Journal of Scientific Study. 2014;2(6):96-100.

15. Jain SK, Jain S, Trikha S. Study of clinical, radiological and bacteriological profile of community acquired pneumonia in hospitalized patients of Garja raja medical college, Gwalior, central India. Int. J. Sci. Study. 2014;2(6):96-100.

16. Bansal S, Kashyap S, Pal LS, Goel A. Clinical and bacteriological profile of community acquired pneumonia in Shimla, Himachal Pradesh. Indian J Chest Dis Allied Sci. 2004;46(1):17-22.

17. Oberoi A, Aggarwal A. Bacteriological profile, serology and antibiotic sensitivity pattern of microorganism from community acquired pneumonia. JK Science. 2006;8(2):79-82.

18. Howard LS, Sillis M, Pasteur MC, Kamath AV, Harrison BD. Microbiological profile of community acquired pneumonia in adults over the last 20 years. J infect. 2005;50(2):107-13.

19. Lee KH, Hui KP, Tan WC, Lim TK. Severe community acquired pneumonia in Singapore. Singapore medical journal. 1996;37:374-77.

20. Ong G, Antonio-Velmonte M, Mendoza MT. Etiologic Agents of Community-Acquired Pneumonia in the Adult: The PGH Experience. Phil J Microbial Infect Dis.1995; 24(2): 29-32.

21. Tadashi I, Toru H, Machiko A, Isao I, Makoto O. Etiology of community acquired pneumonia in hospitalized patients: a 3 year prospective study in Japan. Chest. 1998;114(6):1588-93.

22. Lieberman D, Schlaeffer F, Boldur I, Horowitz S, Friedman MG, Leiononen M, Horovitz O, Mmanor E, Porath A. Multiple pathogens in adult patients admitted with community acquired pneumonia: a one year prospective study of 346 consecutive patients. Thorax. 1996;51(2):179-84.

23. Torres A, Blasi F, Peetermans WE, Viegi G, Welte T. The aetiology and antibiotic management of community acquired pneumonia in adults in Europe: a literature review. Eur J Clin Microbiol Infect Dis. 2014; 33:1065-79.
24. Hamidreza N, Fereshte S, Mohammadreza S, Zahra M, Mehdi JN. Etiological diagnosis of community acquired pneumonia in adult patients. Jundishapur J Microbiol. 2015;8(8):227-80.

25. Fiberesima FP, Onwuchekwa AC. Community acquired pneumonia in Port Hartcourt Rivers state of Nigeria. Cent Afr J Med. 2008; 54(1-4):1-8.

26. Immanuel AM, Faustina P. Prevalence of bacterial pathogens isolated from sputum cultures of hospitalized adult patients with community acquired pneumonia at the Cape Coast Teaching Hospital Ghana. E3 Journal of Medicine Research. 2014; 3(5):58-61.

27. Tao LL, Hu BJ, He LX, Wei L, Xie HM, Wang BG, Li HY, Chen XH, Zhou CM. Etiology and antimicrobial resistance of community acquired pneumonia in adult patients in China. Chin Med J. 2012; 125(17):2967-72.

28. Al-Ghizawi GJ, Al-Sulami A, Hamadi SS. Profile of community and hospital acquired pneumonia cases admitted to basra general hospital, Iraq. East. Mediterr. Health J. 2006; 13(2):230-42.

29. Capoor MR, Nair D, Aggarwal P, Gupta B. Rapid diagnosis of community acquired pneumonia using the BacT/Alert 3D system. Braz J Infect Dis. 2006; 10(5):352-6.

30. Chawla K, Mukhopadhay C, Majumdar M, Bairy I. Bacteriological profile and their antibiogram from cases of acute exacerbations of chronic obstructive pulmonary disease: A hospital based study. J Clin Diagn Res. 2008;2:612-6.

31. Mythri S, Nataraju HV. Bacteriological profile of community acquired pneumonia. IOSR-JDMS. 2013; 12(2):16-9.

32. Bartlett JG, Dowell SF, Mandell LA, File TM Jr, Musher DM, Fine MJ. Practice guidelines for the management of community acquired pneumonia in adults. Infectious Diseases Society of America. Clin Infect Dis. 2000; 31(2):347-82.

33. Singh R, Kour H, Jindal SK, Aggarwal AN, Gupta D, Khan GQ, Magotra S. Global youth tobacco survey: A report from Jammu and Kashmir. JK Science. 2008;10(2):65-9.

34. Nuorti JP, Butler JC, Farley MM, Harrison LH, McGEER A, Kolczak MS, Mreiman RF. Cigarette smoking and invasive pneumococcal disease. N. Eng. J. Med. 2000; 342(10):681-9.

35. Gupta D, Agarwal R, Nath AA, Singh N, Mishra N, Khilnani GC, Samaria JK, Gaur SN, Jindal SK. Guidelines for Diagnosis and Management of Community and Hospital Acquired Pneumonia in Adults: Joint ICS/NCCP (I) Recommendations. Lung India. 2012; 29:S27-S62.

36. Woodhead M, Verheji TJM. A step forward in the everyday management of adults with community acquired pneumonia. BMJ. 2005; 330(7489):460. 